\title{
Portable health monitoring kit using photolethysmogram signal
}

\author{
N.F.A Jamal, K.A. Sidek, A.Z Jusoh \\ Department of Electrical and Computer Engineering, Kulliyah of Engineering, \\ International Islamic University Malaysia, Malaysia
}

\begin{tabular}{l} 
Article Info \\
\hline Article history: \\
Received Oct 9, 2018 \\
Revised Jan 13, 2019 \\
Accepted Mar 2, 2019 \\
\hline
\end{tabular}

\section{Keywords:}

Blood pressure

Heart rate

Portable health monitoring PPG

\begin{abstract}
This study investigates the feasibility of photoplethysmogram (PPG) signals in monitoring health condition and designing a portable health monitoring kit which is suitable for home use. The aim of this study is to ease people in monitoring their health continuously without the need to go to the hospital which can save a lot of time. The focus of this study is to find heart rate and blood pressure recording. The type of PPG sensor used in this project is a non-invasive PPG which measures the blood volume changes in any part of the body. A total of 16 subjects consisting of male and female with age range of 20 to 60 years old were involved in this research. The heart rate and blood pressure for each subject were acquired and analyzed. Based on the result, it shows that higher heart rate reading is associated with female and younger age groups. Meanwhile, for blood pressure value, male subjects tend to have higher blood pressure as compared to female subjects. However, there is no specific pattern for blood pressure in terms of the age group. In the case of HRV analysis based on Kubios software, the SDNN value is higher for male and older age subjects. Meanwhile, the RMSSD value is lower for male and older age subjects. Therefore, PPG signal has the capability to monitor the health status of an individual, which acts as an alternative biological signal for the existing health monitoring systems.
\end{abstract}

Copyright $\odot 2019$ Institute of Advanced Engineering and Science. All rights reserved.

\section{Corresponding Author:}

Nur Firda Ayu Jamal,

Department of Electrical and Computer Engineering,

International Islamic University Malaysia,

P.O. Box 50728 Gombak, Kuala Lumpur.

Email: azami@iium.edu.my

\section{INTRODUCTION}

Nowadays, not many people practice and maintain a healthy lifestyle. This is because some people are not aware about the importance of it. Healthy lifestyle comprises of a wide range of behaviours which includes regular exercise, healthy diet, weight control, tobacco-free lifestyle, weight control, supportive environment and cautious preventive practices. Furthermore, enthusiasm to do regular exercise and sport activities should be nurtured as a study related to physical activities in Malaysia divulged that youth show low interest in exercise and sport activities [1].

There are various steps taken to increase the health awareness among people. One of it is by providing the Ministry of Health Malaysia a community-based data and information associated with health which covers program strategies and activities, health priorities and outlining and allocation of resources. This is done by the Institute for Public Health that conducted the National Health and Morbidity Survey (NHMS) in 2011 for the fourth time. It reported that people in the age range of 18 years old and above, approximately $35.1 \%$ (6.2 million) of them are associated with hypercholesterolemia, $32.7 \%$ (5.8 million) diabetes. Thus, necessary steps and action must be taken to enhance peoples' awareness regarding to their health as most of the people are not concerned with matters related to health. 
One way to deal with this problem is by implementing a portable health monitoring kit. Portable means the size is small and lightweight which can be easily carried from one place to another. Usually, when people feel unwell, they will go to the clinic or hospital. However, sometimes although people are feeling unwell, they will simply prescribed medication from the pharmacy without prior consultation by the doctor. This can lead to several issues because people might not be aware that they have existing health problems. Thus, portable health monitoring kit can help people to check health status from time to time and get useful health information without the need to go to the clinic or hospital. Furthermore, it also can be used by healthy people at any time [3].

Portable health monitoring kit is usually implemented by using the electrocardiogram (ECG) signal due to its simplicity. ECG measures heart rate variability (HRV) and records the electrical activity of the heart [4]-[6]. Meanwhile, HRV measures the variation of the heartbeat in time interval as the rhythm may contain irregularities. This type of monitoring system is usually implemented by doctors at the clinic or hospital. However, for the past few years, a new method in monitoring health status by using PPG signal has been discovered and utilized. A study investigating feasibility of PPG signal by using earlobe PPG to analyze HRV [7] reported that PPG is one of the preferred replacements to ECG. The test was performed by a group of students from Indian Institute of Technology, Delhi by recordings data simultaneously with PPG and HRV and applying the same analytic process for both signals. The results confirmed that PPG provides accurate inter pulse intervals from which HRV measures can be accurately derived in healthy subjects under ideal conditions. Thus, this outcome suggests that health monitoring can be implemented using other biological signals, as an alternative to ECG. In monitoring and checking health status of people, both PPG and ECG signals have their own advantages and features.

PPG shows numerous advantages as compared to ECG such as the presence of both heart rate and respiratory information in the signal. This ability provides an effective PPG-based device to gather both heart rate and respiratory rate information [8] from the same probe. Thus, many health parameters can be obtained by using PPG such as heart rate [9], [10], oxygen saturation and blood pressure. Besides that, PPG has advantageous due to its low cost, lower power consumption [11] and smaller size as compared to ECG acquisition devices. Thus, in this study, a portable health monitoring kit using PPG signal will be developed.

Basically PPG signal measures blood volume changes in any part of the body. This is resulting from the pulsation of blood occurring with each heartbeat. PPG detect cardiovascular waves that propagate through the body using a light source and photo-detector. This study uses a non-invasive method to measure blood volume changes [12] which is a non-contact technique of acquiring bio-signals and is easier to perform. Moreover, this study utilizes a transmission type of PPG sensor which indicates that the light source is placed on the opposite site of the photodetector. PPG signals can be used to assess the cardiovascular conditions [13] because it is directly related to the pulsating arterial blood and synchronous with the heart cycle.

As mentioned earlier, blood volume changes in the microvascular bed of peripheral tissues measured using a PPG sensor originating from a light source and photodetector. Current source is generated according to the amount of reflected light. The light is reflected by the light source which emits light through the skin that reaches the bones. When a raw PPG sensor (a light emitting diode (LED) and a photodetector (PD)) is placed on any part of the body, such as the fingertip, the emitter radiates light into the tissue and the PD will pick up the amount of light that is reflected back from the tissue.

PPG signal waveform consists of two main peaks which are systolic and diastolic. Systolic refers to the end of the cardiac cycle when pressure in the arteries is at its highest or peak. Meanwhile, diastolic is the beginning of the cardiac cycle. This study aims to measure the heart rate and blood pressure of an individual. Heart rate is also called heartbeat. It refers to the number of heart beats per unit time, which is commonly expressed as beats per minute. Heart rate also is a measure of cardiac activity. The heart rate is influenced by various factors such as individual level of physical activity and fitness, condition of body (sleeping, walking, running, resting, etc.), mental and emotional state and circulating hormones. Meanwhile, blood pressure is the force of the blood pushing against the walls of the arteries. Each time the heartbeats, blood will be pumped and pushed into the arteries. Blood pressure is the highest when heartbeats, pumping the blood and thus will reach the highest peak, as has been mentioned earlier which is called systolic blood pressure (SBP). When heart is at rest, between beats and causes blood pressure to fall, this is when diastolic blood pressure (DBP) occurs. SBP and DBP strongly depend on the volume of the blood. Blood pressure below 120/80 is considered as normal meanwhile, blood pressure ranging from 120/80 to $139 / 89$ is called hypertension. SBP is normally around 90 to 100 and when blood pressure is 140/90 or above, it can be considered as high blood pressure [14].

Due to the invention of the ECG signal, it improves the technology for continuous health monitoring system. Nevertheless, alternative method exists with the discovery of PPG signals which is able to record physiological data from almost any part of the body. In such a way, PPG has become better explored and 
utilized, additional applications for the general public have been uncovered but limited only for medical institution implementations. Although, portable health monitoring kit gives many advantages for most of the people, it is still not a commonly used device. As discussed earlier, numbers of researches have been conducted related to the health monitoring system [15] but very few studies have been completed associated to portable device using PPG signals. Hence, the focus of this study is based on a portable device of PPG signal.

Doshi and Panditrao in [16] proposed a hemoglobin (HB) concentration measurement using optical non-contact technique using PPG. The fact that absorption of blood differs at different wavelengths can be used to measure the optical characteristics of blood. Two different wavelengths are set which are $660 \mathrm{~nm}$ and $940 \mathrm{~nm}$. The reason why these two wavelengths are chosen because at 660nm wavelength absorbance of deoxyhemoglobin greatly exceeds the absorbance of oxyhemoglobin whereas at 960nm wavelength absorbance of oxyhemoglobin greatly exceeds the absorbance of deoxyhemoglobin [17], [18]. The objective of this research is to get the heart circulation patterns and light absorbent blood components in the blood by placing a single receiver photo diode in the lower shell of the finger clip.

Jeong et. al, in [19] designed hardware to estimate arterial blood pressure (BP) using PPG signal. Three tests were conducted which are animal, clinical and external factors' tests. Signals were obtained and examined from the tails of three healthy dogs and fingers of six patients who have changes in BP. PPG signal are influenced by pressure of the indexed finger and temperature of the target area. The research was performed to get the relationship of estimation BP from PPG signal. In the animal test, two dogs $20 \mathrm{~kg}$ and $22 \mathrm{~kg}$ were used. The results for animal test show a linear relationship. The clinical tests were processed with patients who were within the range of 9 to 83 years old and were using an invasive blood pressure (IBP) instrument in Wonju Christian Hospital. Meanwhile, for external factors' tests were done by having subjects to take a rest for about 15 minutes and then during the recovery time PPG signal was measured. From this study, it concluded that the blood volume is proportional to temperature and inversely proportional to pressure.

Gil et. al, in [20] investigated the pulse transmit time variability with heart rate variability during decrease in the amplitude fluctuations of PPG signal. Pulse transit time (PTT) can be measured by using PPG because it is directly related with cardiac function [21]. The main objective to analyze the transmitted pulse is to compare the PTT variability with HRV during amplitude fluctuations of PPG events. They also analyzed the relationship with sleep apnea. Hence from their analysis, they can improve specificity of amplitude fluctuations of PPG as apnea market. From the analysis, it stated that PTT reflected sympathetic changes more obvious than HRV. It is because PPT reflects peripheral circulation which mainly is mediated by sympathetic activity.

Although there are many research on PPG signal related to health monitoring system, however, portable health monitoring kit has been yet uncovered. Portability creates accessibility to the general public. Thus, in this research, we will develop a portable health monitoring kit using PPG signal.

\section{RESEARCH METHOD}

This section consists of two main parts which are; i) Proposed Methodology and ii) Hardware Design in developing portable health monitoring kit using PPG signal.

\subsection{Proposed Methodology}

In this study, four steps are done to develop and implement a portable health monitoring kit using PPG signal which consists of signal acquisition, preprocessing, feature extraction and classification.

\subsubsection{Signal Acquisition}

The PPG signals were acquired using a sensor module called Easy Pulse v1.1 which is connected to the Arduino microcontroller. A total of 16 subjects which consist of male and female with age range from 20 to 60 years olds are involved in this research. Note that, different subjects will give diverse PPG signal based on the level of their health, size, age and also gender. These signals were acquired by using IR LED sensor as the light source and the phototransistor as the light detector. In this study, these sensors were applied on the fingertip. In the next stage, the acquired signal will go undergo preprocessing to remove noise of the signal.

\subsubsection{Preprocessing}

The light that hits the skin and passing through the tissue is measured by a photodetector which produces a signal. Note that the measured signals can be different and distorted by various ambient noise. The PPG signal obtained from the photodetector is noisy and weak. Thus, it is required to have an amplifier to boost the signal and a filter to remove unwanted portion of the signal. In the first stage, the signal will pass 
into a passive high pass filter to further block any DC components. The output signal from the high pass filter then will go to a low pass filter. In the next stage, there are also additional high pass and low pass filters and the output obtained from those steps will be fed to an Operational Amplifier (OP Amp) [22].

\subsubsection{Feature extraction}

In this project, we basically used Arduino only to find the health parameters due to the requirement of portability. Once the signal has been filtered, discriminant features were extracted. One cycle of PPG (which consist of systole and diastole region) signal was extracted and analyzed to obtain the desired health parameters as shown in Figure 1. The PPG waveform contains important features needed to analyze the signal. The peak detection and alternating current (AC) components value are essential in measuring heart rate. Furthermore, blood pressure measurements require crucial calculations which the shape and variations of PPG signal will affect the readings. After obtaining the extracted features, it will then act as the input for the proposed classification mechanisms.

\subsubsection{Signal Classification}

We use statistical analysis to classify the signal. There are different formulas to calculate each important parameter. In the study, standard algorithms were used in order to obtain the readings which are heart rate and blood pressure. In this algorithm the PTT and pulse wave velocity (PWV) are important in determining the heart rate and blood pressure. PTT as shown in Figure 2 refer to as the time it takes the pulse wave to travel between two arterial sites within the same cardiac cycle. This time is related to the propagation velocity of the pulse wave [21]. Meanwhile PWV is the speed at which the arterial pressure wave travels is directly proportional to blood pressure [21]. The pulse wave velocity is measured by measuring PTT, which refers to the time interval between two pulse waves propagating in the same cardiac cycle from separate arterial sites [21]. After obtaining PTT and PWV, these values will be added to the specified formulas. These formulas are briefly described as in (1), (2) and (3) in reference to [21].

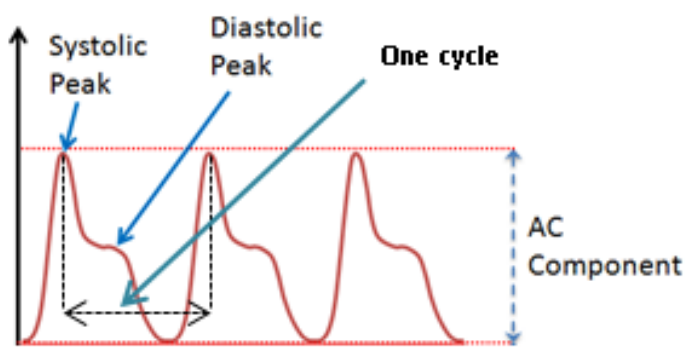

Figure 1. One cycle of PPG signal [22]

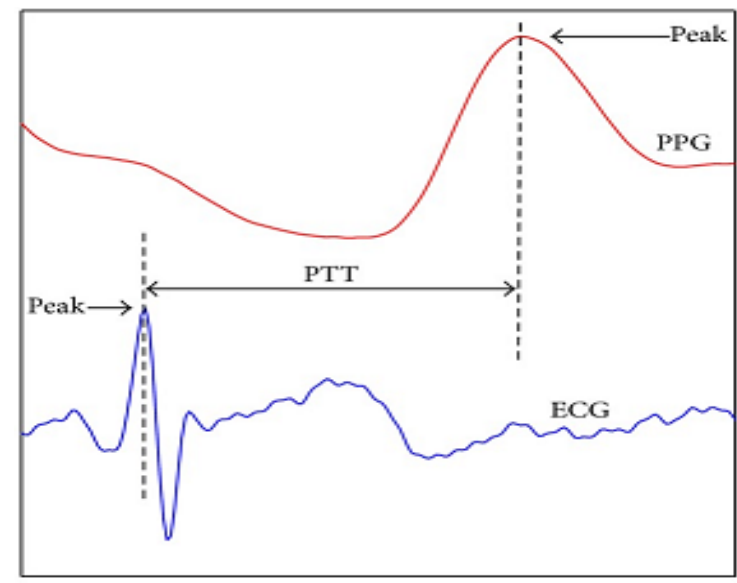

Figure 2. PTT of PPG and ECG signal [23]

$$
\begin{aligned}
& \text { Heart Rate }=f \times 60 \text { or }(2 \times 60) \div(P T T 1+P T T 2) \\
& S B P=0.05089855 \times P W V+62.5590972 \\
& D B P=0.04940772 \times P W V+17.4800472
\end{aligned}
$$

where: $\mathrm{f}=$ frequency, $\quad$ PTT $=$ Pulse transit time, $\quad$ PWV $=$ Pulse wave velocity of the signal

When obtaining the heart rate and blood pressure, the points for the PPG signals also must be acquired for the analyzation and comparing purposes. This type of analysis is specifically for heart rate measurement. We used Kubios HRV software in the analyzation of the points of PPG signal for efficient and accurate comparison.

Kubios HRV is an advanced and simple to utilize programming for HRV examination. The product underpins a few information groups for PPG or ECG information and beat-to-beat RR interim information. 
In this research, the focus is on the time-domain analysis. Due to the short term of the data statistics which is less than 10 minutes, the time-domain involved are standard deviation of normal to normal intervals (SDNN) and root mean square of successive differences (RMSSD) on normal to normal (NN) intervals.

SDNN is very significant and important because it reflects the factors that contribute to the heart rate variability including the slow oscillations which are believed to reflect the heart's intrinsic ability to respond to hormonal influences. A variance is mathematically equivalent to the total power of spectral analysis, so it reflects all cyclic components of the variability in recorded series of NN intervals. The length of recording affects the actual values of SDNN because these values are proportional to the length of the recording. Moreover, SDNN also determine the stress coping ability of an individual. Meanwhile, RMSSD measures the estimated high-frequency variations in heart rate in short term $\mathrm{NN}$ recordings which reflect an estimate of parasympathetic regulation of the heart. Note that RMSSD is measured in milliseconds.

\subsection{Hardware Design}

The hardware components used for this project are Arduino Mega 2560, Easy Pulse Sensor v1.1, thin flim transistor (TFT) touch screen and power source. Each of this hardware will be briefly described in this section.

\subsubsection{Arduino Mega 2560}

Arduino Mega 2560 is one of the compact and powerful microcontroller board based on the ATmega2560. It contains 54 digital input/output pins, 16 analog inputs, 4 UARTs (hardware serial ports), a $16 \mathrm{MHz}$ crystal oscillator, a Universal Serial Bus (USB) connection, a power jack, an In-Circuit Serial Programming (ICSP) header, and a reset button. It is responsible to support the microcontroller because it contains various things needed. In order to get started, simply connect it to a computer with a USB cable or power it with battery or an AC-to-DC adapter. The Arduino Mega 2560 board is compatible with most shields designed for the Uno and the former boards Duemilanove or Diecimila.

\subsubsection{Easy Pulse Sensor v1.1}

Easy Pulse Sensor v1.1 is used to acquire the PPG signal from the fingertip. It consists of IR LED and photo detector. This sensor makes use of transmission PPG which the IR LED and phototransistors are placed oppositely to each other. This type of sensor is susceptible to the small movement of the finger. In order to get an accurate pulse of PPG signal, finger must be very steady and avoid noticeable movement. Moreover, Easy Pulse Sensor v1.1 uses an improve sensor type which also known as HRM-2155E that is less prone to the finger motion.

\subsubsection{TFT Touch Screen}

In this project, 2.4" TFT touch screen was used to display the outputs. This type of TFT display is 2.4" diagonal with 4 white-LED backlight. The display is $240 \times 320$ pixels with individual Red Green Blue (RGB) pixel control, which the resolution is good than the normal black and white 128x64 display. Moreover, the TFT touch screen comes with resistive touch screen in order to detect finger presses in any point on the screen. The advantage of this touch screen is it has its own controller with RAM buffering which causes the microcontroller to remain idle almost all the time. The display can be used in two modes, which are 8-bit and SPI.

For 8-bit mode, there are 8 digital data lines and 4 or 5 digital control lines to read and write to the display (12 lines total). Meanwhile, SPI mode requires only 5 pins total (SPI data in, data out, clock, select, and $\mathrm{d} / \mathrm{c}$ ) but is slower than the 8-bit mode.

\subsubsection{Power Source}

Power source is needed in order to supply the power to the embedded system. This is due to the proposed health monitoring kit which offers portability and wireless criterion. A power bank is used as the power source with $5 \mathrm{~V}$ output voltages and $1 \mathrm{~mA}$ current. It is very important to know the maximum and minimum limit of the current and voltage that are used to operate the device in order to avoid any damages or faults that might occur if the power source limit exceeds the required range.

\section{RESULTS AND ANALYSIS}

This section will discuss the experimentation procedure and perform the result analysis based on heart rate and blood pressure. In order to get the desired output, several steps must be done in developing the portable health monitoring kit. PPG signal can be obtained directly from the finger of subject by using Easy Pulse v1.1. In this research, at the beginning of the data acquisition, subjects were required to be in a sitting 
and relaxed condition to obtain the desired waveforms. This is due to any movement of the subjects will affect the output. Subjects need to calm down for about 5 minutes before PPG signals can be collected. Furthermore, Subjects can read their health parameters through the TFT touchscreen.

\subsection{Portable Healts Monitoring Kit Display Screen}

When the device starts, the welcoming page will appear and the user will be asked to place his/her finger on the sensor. Then, the device will prompt for user's height and display a keypad containing numbers on the TFT touch screen. Thus, the user must key in the estimated height by pressing the numbers on the TFT touchscreen. User must wait for a while before the device can display the heart rate and blood pressure values.

\subsection{Result Analysis}

A total of 16 subjects were classified based on gender and age. There are four age ranges that were considered which are 20 to 30,30 to 40,40 to 50 and 50 to 60 years old. The heart rate and blood pressure for the 16 subjects are shown in Table 1.

Table 1. Heart Rate and Blood Pressure of 16 Subjects

\begin{tabular}{ccccc}
\hline Subject & Gender & Age & $\begin{array}{c}\text { Heart Rate } \\
(\text { bpm })\end{array}$ & $\begin{array}{c}\text { Blood Pressure } \\
\text { (Systolic/ Diastolic) }\end{array}$ \\
\hline 1 & Female & $20-30$ & 97 & $100 / 66$ \\
2 & Female & $20-30$ & 84 & $108 / 70$ \\
3 & Male & $20-30$ & 74 & $114 / 76$ \\
4 & Male & $20-30$ & 122 & $128 / 89$ \\
5 & Female & $30-40$ & 80 & $112 / 75$ \\
6 & Female & $30-40$ & 78 & $106 / 69$ \\
7 & Male & $30-40$ & 80 & $114 / 75$ \\
8 & Male & $30-40$ & 64 & $106 / 71$ \\
9 & Female & $40-50$ & 90 & $113 / 76$ \\
10 & Female & $40-50$ & 79 & $120 / 82$ \\
11 & Male & $40-50$ & 66 & $117 / 82$ \\
12 & Male & $40-50$ & 78 & $113 / 74$ \\
13 & Female & $50-60$ & 70 & $101 / 64$ \\
14 & Female & $50-60$ & 66 & $101 / 64$ \\
15 & Male & $50-60$ & 59 & $99 / 64$ \\
16 & Male & $50-60$ & 63 & $106 / 69$ \\
\hline
\end{tabular}

As a result of the experimentation procedure, the heart rate and blood pressure for various age ranges and genders are different as seen in Table 1. The ranges for heart rate vary from $59 \mathrm{bpm}$ to $100 \mathrm{bpm}$. However, there are two male subjects that show slightly unusual reading for heart rate which are Subject 4 and 15. For Subject 4, the heart rate is $122 \mathrm{bpm}$ which is considered as high that is due to the emotion or physical condition of the subject when the data acquisition took place as the subject is only in the age range of 20 to 30 years old. On the other hand, Subject 15 showed a relatively lower heart rate which is 59 bpm. The subject was asked if he has any history of heart abnormality in order to get further details. The subject mentioned that he is under continuous medication and usually his heart rate is around $60 \mathrm{bpm}$ as measured by the doctor. Hence, our proposed heart rate measurement is synchronized with the clinical reading. Furthermore, blood pressure values also show different reading ranging from 99/64 to 120/82 except for one subject. The subject with the highest heart rate gave the highest blood pressure which is $128 / 89$. The possible reason of this situation is due to anxiety which causes physical changes such as increased the blood pressure and heart rate.

Heart rate and blood pressure can be further classified and analyzed based on gender and age ranges to get significant differences. Table 2 shows the heart rate statistical analysis based on genders. From Table 2, it can be observed that the average heart rate for female subjects is higher than male subjects by approximately $5 \mathrm{bpm}$. Although female subjects show a relatively higher reading for the average heart rate, however, the range for the heart rate is still in the normal range. Furthermore, the standard deviation of the heart rate for male subjects is larger as compared to the female subjects. This implies that the heart rate of male subjects is spread over out of the mean range wider than female subjects. By comparing the gender differences, male subjects tend to have slower heart rate. This is because male subjects tend to be more relaxed and calmed as compared to the female subjects. 


\begin{tabular}{cccc}
\multicolumn{3}{c}{ Table 2. Heart Rate Statistical Analysis based on Genders } \\
\hline Subjects & $\begin{array}{c}\text { Sum } \\
\text { (bpm) }\end{array}$ & $\begin{array}{c}\text { Average } \\
(\mathrm{bpm})\end{array}$ & Standard Deviation \\
\hline Both male and female & 1250 & 78.125 & 15.569 \\
Male & 606 & 75.75 & 20.148 \\
Female & 644 & 80.5 & 10.029 \\
\hline
\end{tabular}

After categorizing the subjects according to the gender, the analysis on the age range is considered. As mentioned earlier, the subjects are divided into four age groups. The statistical analysis of heart rate for each age group is shown in Table 3.

Table 3. Statistical Analysis of Heart Rate According to Age Groups

\begin{tabular}{cccc}
\hline Age (years old) & Sum $(\mathrm{bpm})$ & Average (bpm) & Standard Deviation \\
\hline $20-30$ & 377 & 94.25 & 20.759 \\
$30-40$ & 302 & 75.50 & 7.724 \\
$40-50$ & 313 & 78.25 & 9.811 \\
$50-60$ & 258 & 64.50 & 4.655 \\
\hline
\end{tabular}

The sum and average heart rate for the age group of 20 to 30 years old shows the highest reading with $377 \mathrm{bpm}$ and $94.25 \mathrm{bpm}$ respectively. Meanwhile, the age group of 50 to 60 years old has the lowest sum and average heart rate. This is because younger age groups are more active in term of physical activities as compared to older age groups. There is a huge difference in the sum and average heart rate for these two age groups. The results show that older people tend to have lower heart rate as compared to younger people. In addition, the standard deviation for each age group is proportional to their sum and average heart rate. This specifies that the higher the sum and average heart rate, the higher the standard deviation.

Blood pressure also can be categorized in more details according to gender. Table 4 shows a statistical analysis of blood pressure based on gender.

Table 4. Statictical Analysis of Blood Pressure based on Gender

\begin{tabular}{ccc}
\hline Subject & \multicolumn{2}{c}{ Average } \\
\cline { 2 - 3 } & SBP & DBP \\
Both male and female & 109.875 & 72.875 \\
Male & 112.125 & 75 \\
Female & 107.625 & 70.75 \\
\hline
\end{tabular}

The average SBP and DBP of both male and female subjects are in the normal range. However, SBP and DBP of male subjects are higher as compared to female subjects. The reason of this result is due to the weight of the subjects. According to the research conducted by Harsha and Bray [13], blood pressure alteration is theorized to be positively associated with weight change. This can be explained as by the reductions in insulin resistance, enhanced sodium retention, alterations in vascular structure and function, changes in ion transport, enhanced stimulation of the renninaldosterone-angiotensin system, increased activation of sympathetic nervous system, and changes in natriuretic peptide when weight is reduce. Thus, a healthy diet should be maintained because it will affect the blood pressure. Besides that, the standard deviation of male subjects also tends to deviate more than female subjects. In addition, the blood pressure also has been analyzed statistically according to age groups as tabulated in Table 5 .

Table 5. Statictical Analysis of Blood Pressure According to Age Groups

\begin{tabular}{ccccc}
\hline Age & \multicolumn{2}{c}{ Average } & \multicolumn{2}{c}{ Standard Deviation } \\
\cline { 2 - 5 } (years old) & SBP & DBP & SBP & DBP \\
$20-30$ & 112.5 & 75.25 & 11.818 & 10.046 \\
$30-40$ & 109.5 & 72.5 & 4.123 & 3 \\
$40-50$ & 115.75 & 78.5 & 3.403 & 4.123 \\
$50-60$ & 101.75 & 65.25 & 2.986 & 2.5 \\
\hline
\end{tabular}


According to age groups, the SBP and DBP for 20 to 30 years old and 40 to 50 years old have higher reading as compared to the remaining age groups. In contrast, subjects in the age of 50 to 60 years old have the lowest SBP and DBP. The reason being is due to the medication effect and also the stress level. The subjects in the age range of 40 to 50 years old are more likely to have higher level of stress as compared to the other age group. Many factors can contribute to the stress level such as the environment and personal issues. Although the subjects in the age group of 40 to 50 years old have the highest blood pressure, however, the standard deviation for both SBP and DBP is not high as compared to the subjects in the age group of 20 to 30 years old. This shows that the blood pressure of the subjects in the age of 20 to 30 years old have a large difference with each other.

Overall, from the above analysis, it can be concluded that for the heart rate, female and younger age group have higher heart rate as compared to others groups. Meanwhile, for blood pressure, male subjects have higher blood pressure as compared to female subjects. However, according to age groups, the blood pressure does not follow a specific pattern. The subjects in the age group of 50 to 60 have the lowest blood pressure and subjects in the age groups of 40 to 50 have the highest blood pressure.

\subsection{HRV Analysis}

As been mentioned earlier, time-domain analysis will be performed on the HRV. The results from Kubios software for 1 male subject (in the age range of 20 to 30 years old) and 1 female subject (in the age range of 40 to 50 years old) are shown in Figure 3 and 4 respectively.

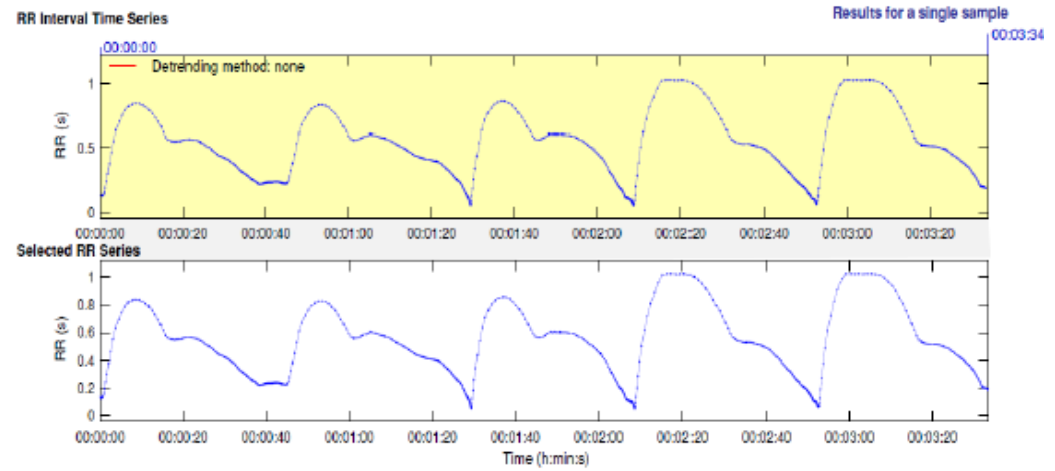

Figure 3. Results from Kubios software for male subject in the age range 20 to 30 years old

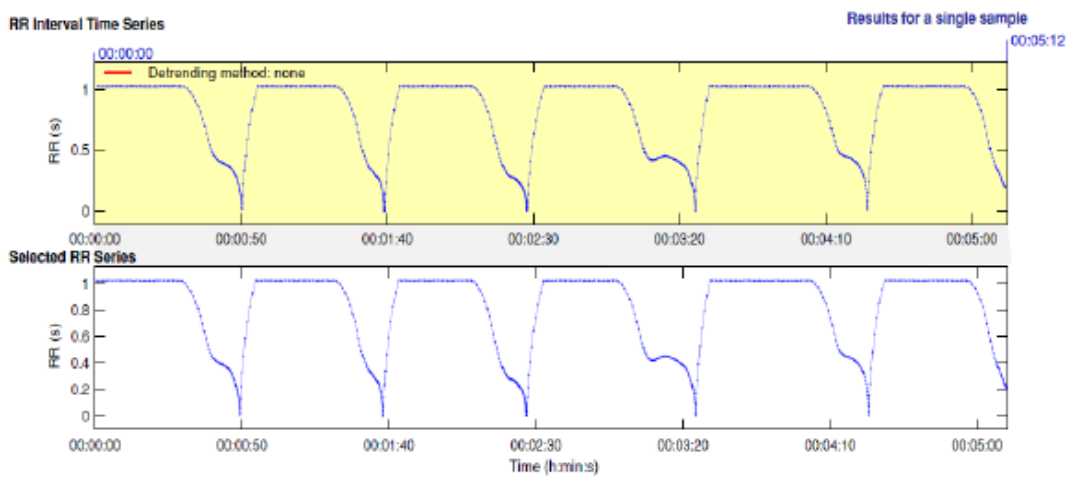

Figure 4. Results from Kubios software for female subject in the age range 40 to 50 years old

Based on Figure 3 and 4, it can be seen that each subject has their own pattern of PPG signals. There are many factors that affect the waveform of PPG signal such as age, gender, physical condition and etc. The variation of PPG signal lead to variation of heart rate variability. By using Kubios software, important information can be obtained and analyzed. Only two time-domain measurements will be highlighted in this study which is SDNN and RMSSD. The subjects' SDNN and RMSSD are shown in Table 6. 
From Table 6, according to the age ranges, the SDNN is higher at the age of 50 to 60 years old. This can be seen clearly as all of the subjects in the age range of 50 to 60 years old have SDNN that are greater than $300 \mathrm{~ms}$. Meanwhile, for the age range of 20 to 30 years old, one of the subjects shows the lowest SDNN with $250.8 \mathrm{~ms}$. The other subjects in similar age range gave a common SDNN measurement. In addition, subjects in the age range of 30 to 40 and 40 to 50 years old show the same trend, where mostly the subjects have SDNN in the range of $270 \mathrm{~ms}$ to $288 \mathrm{~ms}$. These show us that as age increases, the SDNN also increases. Thus, low SDNN is associated with low HRV, which primarily indicates the reduction in dynamic complexity. Based on the experimentation, as age increases, the stress coping ability is good because of the higher SDNN value. Lower SDNN shows that there is a risk of developing stress induced disease, which weakens the autonomic nervous system (ANS) [25].

\begin{tabular}{ccc}
\multicolumn{3}{c}{ Table 6. SDNN and RMSSD of 16 Subjects } \\
\hline Subject & SDNN $(\mathrm{ms})$ & RMSSD $(\mathrm{ms})$ \\
\hline 1 & 290.1 & 99.9 \\
2 & 332 & 45.6 \\
3 & 307.3 & 44.8 \\
4 & 250.8 & 28.2 \\
5 & 297.7 & 28.9 \\
6 & 323.2 & 41.3 \\
7 & 387.1 & 57.8 \\
8 & 298.9 & 32.2 \\
9 & 293.7 & 76.6 \\
10 & 359.3 & 38.3 \\
11 & 360.8 & 43.7 \\
12 & 277.5 & 36.4 \\
13 & 305.4 & 51.3 \\
14 & 337.3 & 49.5 \\
15 & 324.9 & 37.9 \\
16 & 359.2 & 48.5 \\
\hline
\end{tabular}

This outcome is in line with the result in Table 4.10 where the subjects in the age group of 50 to 60 years old have lowest level of stress due to their low blood pressure. Thus, as the age approaches the retirement phase of 60 years old an individual may feel less stressful due to surrounding factors. Furthermore, in Table 5, the age range of 20 to 30 years old has the lowest SDNN which is due to the samples acquired from the students in the university. The cause of this situation may be due to multiple assignment and examination. As a result, they tend to have lower coping ability to various emotional and physical stressors.

The average RMSSD for the age class of 20 to 30 years old is $54.63 \mathrm{~ms}, 40.1 \mathrm{~ms}$ for the age class of 30 to 40 years old, $48.75 \mathrm{~ms}$ for the age class of 40 to 50 years old and $46.8 \mathrm{~ms}$ for the age class of 50 to 60 years old. It can be deduced that as the age increases, ironically the RMSSD decreases. This parameter is associated with the electrical stability of heart which is influenced by the parasympathetic nervous system (PNS) activity. It is due to as age increased and hence RMSSD decreased where there is a greater risk of developing cardiac disease [25].

The SDNN and RMSSD can also be analyzed in terms of the gender difference as shown in Table 7. Table 7 shows that the average SDNN for male subjects is greater as compared to female subjects. This is not surprising as male tends to be relaxed and not easily affected by emotion. It also indicates that male subjects have a good stress coping ability as compared to female subjects. Meanwhile, the average RMSSD for male subjects is lower than the female subjects. It reflects that male subjects have higher chance of attaining cardiac abnormalities and relatively weak PNS. The possible contributing factors of this behavior may be due the smoking habit of some of the male subjects and as we are aware that this habit will definitely affect our health in general.

Based on the above analysis, it shows that according the age range, SDNN is higher for older age range. Besides that, according to gender, male subjects have higher SDNN as compared to female subjects. Meanwhile, the RMSSD is lower when age increases. In addition, male subjects also have lower RMSSD than female subjects. 


\begin{tabular}{|c|c|c|c|c|}
\hline & \multicolumn{2}{|c|}{$\begin{array}{c}\text { SDNN } \\
(\mathrm{ms})\end{array}$} & \multicolumn{2}{|c|}{$\begin{array}{l}\text { RMSSD } \\
(\mathrm{ms})\end{array}$} \\
\hline & Male & Female & Male & Female \\
\hline & 307.3 & 290.1 & 44.8 & 99.9 \\
\hline & 250.8 & 332 & 28.2 & 45.6 \\
\hline & 387.1 & 297.7 & 57.8 & 28.9 \\
\hline & 298.9 & 323.2 & 32.2 & 41.3 \\
\hline & 360.8 & 293.7 & 43.7 & 76.6 \\
\hline & 277.5 & 359.3 & 36.4 & 38.3 \\
\hline & 324.9 & 305.4 & 37.9 & 51.3 \\
\hline & 359.2 & 337.3 & 48.5 & 49.5 \\
\hline Average & 320.8125 & 317.3375 & 41.1875 & 53.925 \\
\hline
\end{tabular}

\section{CONCLUSION}

To conclude, all of the objectives for this research have been achieved successfully. The proposed health monitoring kit managed to obtain user heart rate and blood pressure with a portability criterion. This project also was installed with a TFT touch screen which enhanced the GUI capability as compared to liquid crystal display (LCD) in assisting user when utilizing this health monitoring kit. Moreover, from this research, it shows that different subjects will have different heart rate and blood pressure reading according to the gender, age, physiological condition and etc. Besides that, based on the HRV analysis on Kubios software, SDNN value is higher for male and older age subjects. Meanwhile, the RMSSD value is lower for male and older age subjects. In addition, our findings suggest that PPG signal is one of the preferred alternatives to ECG signal in acquiring health parameters. Lastly, this research highlighted that by using a portable health monitoring kit, early and undetected signs of signal abnormalities of a person has the potential to save more life and at the same time, ease people in monitoring their health status faster and efficiently.

This study has been emphasized in developing a portable health monitoring kit by using PPG signal that can help people in many aspects of life. Perfection is a dominant target in every development of technology. However, every technology produced will have limitations. Our portable health monitoring kit has some inadequacies which are the TFT touch screen is not clear and user is not able to estimate how long the device can operate from the touch screen.

Therefore, in the future, the other model type of TFT touch screen can be used to get a clear interface. This includes larger size of TFT touch screen in order to get a better resolution. Besides that, the system also can be equipped with a battery percentage indicator on the screen to determine how long the device can operate to ensure a flawless flow of the device.

\section{ACKNOWLEDGEMENT}

First and foremost, I am grateful to Allah SWT for giving me the health and strength to complete my Final Year Project (FYP) report. I would like to express my deepest gratitude and appreciation towards my highly motivated and kind supervisor, Dr. Khairul Azami Sidek for his constant support, courage, motivation and words of wisdom throughout the semester. It was a pleasure to work under his supervision.

Besides that, I want to extend my appreciation to the FYP Coordinator, Dr. Suriza Ahmad Zabidi for her guidance regarding the final year project. Last but not least, special thanks to my family, friends and whoever are involved in completing my project. May Allah reward all of them for their priceless kindness and support.

\section{REFERENCES}

[1] M. N. Othman, S. F. Yap and Y.G. Wee, "Examining the Relationship between Gender, Age, Education Level and Social Cognitive Factors in a Health Setting." International Journal of Business and Management, Vol. 6, No. 9, p.2, September 2011.

[2] M. L. Sahu and J. K. Kaushal, "Real time health monitoring system using Arduino and LabVIEW with GSM Technology," pp. 3-7.

[3] R. Bhimanpallewar, S. Patil, S. Kambale, and R. Kedari, "Wireless Healthcare Monitoring System using Arduino Uno," vol. 4, no. 12, pp. 604-607, 2017.

[4] D. C. Dugdale and D. Zieve, "Electrocardiogram.” MedlinePlus Medical Encyclopaedia, p.3, 2012.

[5] M. A. H. Assan, A. S. M. Alik, D. F. Ofi, N. S. Aad, and M. Eriaudeau, "Novel health monitoring method using an RGB camera," Biomed. Opt. Express, vol. 8, no. 11, p. 4838, 2017.

[6] B. M. Jayadevappa and M. S. Holi, "An Estimation Technique using FFT for Heart Rate Derivedfrom PPG Signal," vol. 15 , no. $7,2015$. 
[7] N. Selvaraj, A. Jaryal, J. Santhosh, K. K. Deepak and S. Anand, "Assessment of heart rate variability derived from finger-tip photoplethysmography as compared to electrocardiography." Centre for Biomedical Engineering, Indian Institute of Technology-Delhi, 32(6): 479-84, 2008 Nov-Dec.

[8] S. Li, L. Liu, J. Wu, B. Tang, and D. Li, "Comparison and Noise Suppression of the Transmitted and Reflected Photoplethysmography Signals,” vol. 2018, 2018.

[9] S. Kavitha, M. ArulPugazhendhi, and B. Vinodh Kumar, "VLSI Implementation of PPG Signal for Health Monitoring System,” Int. J. Comput. Sci. Mob. Comput., vol. 5, no. 3, pp. 667-675, 2016.

[10] S. C. Mukhopadhyay, "Wearable Sensors for Human Activity Monitoring : A Review," no. December, 2015.

[11] C. Orphanidou, T. Bonnici, P. Charlton, D. Clifton, D. Vallance, and L. Tarassenko, "Signal-Quality Indices for the Electrocardiogram and Photoplethysmogram : Derivation and Applications to Wireless Monitoring," vol. 19, no. 3, pp. 832-838, 2015.

[12] A. A. Kamshilin and N. B. Margaryants, "Origin of photoplethysmographic waveform at green light," Phys. Procedia, vol. 86, no. June 2015, pp. 72-80, 2017.

[13] S. Assad, F. Ding, N. Fu and Y. Joy Xu, "Correlating Heart Rate Variability with Mental Fatigue." p.22, April 26, 2012.

[14] High Blood Pressure Symptoms, Causes and Treatments, (Accessed on 30 $0^{\text {th }}$ March 2015) http://www.medicinenet.com/high_blood_pressure_pictures_slideshow/article.html

[15] S. G. Khalid, J. Zhang, F. Chen, and D. Zheng, "Blood Pressure Estimation Using Photoplethysmography Only: Comparison between Different Machine Learning Approaches," vol. 2018, 2018.

[16] R. Doshi and A. Panditrao, "Non-Invasive Optical Sensor for Hemoglobin Determination." International Journal of Engineering Research and Applications (IJERA), Vol. 3, Issue 2, pp.559-562, March -April 2013.

[17] H. P. Brecht, PetrovBrecht, "Noninvasive continuous optoacoustic monitor of total hemoglobin concentration." Engineering in Medicine and Biology Conference, Vol. 3, 2002.

[18] S. G. Khalid, J. Zhang, F. Chen, and D. Zheng, "Blood Pressure Estimation Using Photoplethysmography Only: Comparison between Different Machine Learning Approaches," vol. 2018, 2018.

[19] C. Jeong, J. Ko, S. Hwang, and H. Yoon, "A New Method to estimate Arterial Blood Pressure using Photoplethysmographic Signal." 28th IEEE EMBS Annual International Conference New York City, USA, Aug 30Sept 3, 2006.

[20] E. Gil, J. Vergara, and P. Lagun, "Pulse Transit Time Variability versus Heart RateVariability during Decreases in the Amplitude fluctuations of Photoplethysmography signal." International Journal of Bioelectromagnetism, Vol. 12, No. 3, pp. 95 - 101, 2010.

[21] S. Golil and Jayanthi, "Cuff less Continuous Non-Invasive Blood Pressure Measurement Using Pulse Transit Time Measurement." International Journal of Recent Development and Technology, Vol. 2, Issue 1, pp. 86-91, 2014.

[22] Embedded Lab, "Introducing Easy Pulse: A DIY photoplethysmographic sensor for measuring heart rate Embedded Lab", 2012. (Accessed on $2^{\text {nd }}$ January 2016) http://embedded-lab.com/blog/easy-pulse-version-1-1sensor overview-part-1/

[23] A Blood Pressure Monitoring Method for Stroke Management (Accessed on $5^{\text {th }}$ October 2018) https://www.researchgate.net/figure/Pulse-transit-time-is-defined-as-the-time-interval-between-the-R-peak-ofECG-and-the-peak_fig1_265418109

[24] D. Harsha and G. Bray, "Weight Loss and Blood Pressure Control (Pro)", Hypertension, vol. 51, no. 6, pp. 1420$1425,2008$.

[25] SA-3000P Clinical Manual VER.3.0: Heart Rate Variability Analysis System, MEDICORE Co., Seoul, Korea, (online). Available: http://medi-core.com/download/HRV_clinical_manual_ver3.0.pdf

\section{BIOGRAPHIES OF AUTHORS}

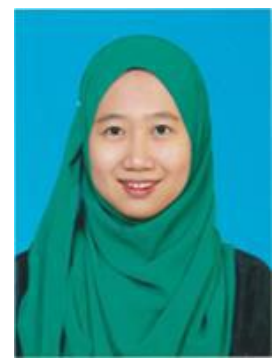

Nur Firda Ayu Jamal graduated Bachelor's degree in Communication Engineering at International Islamic University Malaysia (IIUM) in 2016.Currently, she is taking Master in Science (Communication Engineering) from the same university. She has experience about one year in industrial before she started her Master's Degree. Her area of interest is in the field of biomedical signal processing, wireless and satellite communication. 

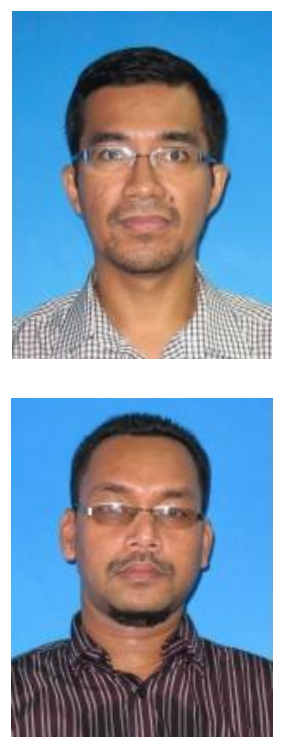

Dr. Khairul Azami Sidek, a graduate of the International Islamic University Malaysia (IIUM), started his career as an Assistant Lecturer at the Department of Electrical and Computer Engineering, Kulliyyah of Engineering, IIUM in 2004. In 2007, he was appointed as a lecturer in the same department after completing his Master's degree in University Kebangsaan Malaysia. Later, in 2014, he was appointed as an Assistant Professor after finishing his PhD studies in RMIT University, Melbourne, Australia. Recently, in August 2018, he was promoted to the post of Associate Professor in the same departm ent. His area of interest is in the field of biometric recognition, pattern recognition and biomedical signal processing.

Dr. Ahmad Zamani Jusoh, completed his Bachelor's degree at the Hanyang University. He has obtained his Master's degree and $\mathrm{PhD}$ in Communication Engineering from University of Loughborough and University of Western Australia, respectively. Currently he is an Assistant Professor at Electrical and Computer Engineering Department, International Islamic University Malaysia (IIUM). His area of interest are speech signal processsing and digital communication networks. 